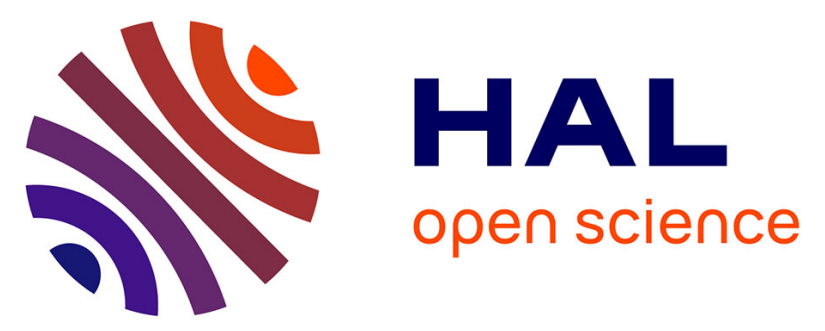

\title{
Low Energy Characterization of Caliste HD, a Fine Pitch CdTe-Based Imaging Spectrometer
}

Sebastien Dubos, Olivier Limousin, Claire Blondel, Remi Chipaux, Youri

Wladimir Dolgorouky, Olivier Gevin, Yves Ménesguen, Aline Meuris, Thierry

Orduna, Thierry Tourette, et al.

\section{To cite this version:}

Sebastien Dubos, Olivier Limousin, Claire Blondel, Remi Chipaux, Youri Wladimir Dolgorouky, et al. Low Energy Characterization of Caliste HD, a Fine Pitch CdTe-Based Imaging Spectrometer. IEEE Transactions on Nuclear Science, 2013, 60 (5), pp.3824 - 3832. 10.1109/TNS.2013.2281572 . cea-01791971

\section{HAL Id: cea-01791971 https://hal-cea.archives-ouvertes.fr/cea-01791971}

Submitted on 18 Feb 2019

HAL is a multi-disciplinary open access archive for the deposit and dissemination of scientific research documents, whether they are published or not. The documents may come from teaching and research institutions in France or abroad, or from public or private research centers.
L'archive ouverte pluridisciplinaire HAL, est destinée au dépôt et à la diffusion de documents scientifiques de niveau recherche, publiés ou non, émanant des établissements d'enseignement et de recherche français ou étrangers, des laboratoires publics ou privés. 


\section{Low Energy characterization of Caliste HD, a fine pitch CdTe-based Imaging Spectrometer}

\begin{tabular}{|c|c|}
\hline Journal: & IEEE Transactions on Nuclear Science \\
\hline Manuscript ID: & Draft \\
\hline Manuscript Type: & RTSD \\
\hline Date Submitted by the Author: & $n / a$ \\
\hline Complete List of Authors: & $\begin{array}{l}\text { Dubos, Sébastien; CEA Saclay, DSM/IRFU/SAp } \\
\text { Limousin, Olivier; CEA Saclay, DSM/IRFU/SAp } \\
\text { Blondel, Claire; CEA Saclay, DSM/IRFU/SAp } \\
\text { Chipaux, Remi; CEA, DSM/IRFU/SEDI } \\
\text { Dolgorouky, Youri; APC, } \\
\text { Gevin, Olivier; CEA Saclay, DSM/IRFU/SEDI } \\
\text { Ménesguen, Yves; CEA Saclay, DRT/LIST/LNHB } \\
\text { Meuris, Aline; CEA Saclay, DSM/IRFU/SAp } \\
\text { Orduna, Thierry; CEA Saclay, DSM/IRFU/SAp } \\
\text { Tourette, Thierry; CEA Saclay, DSM/IRFU/SAp } \\
\text { Sauvageon, Aymeric; CEA Saclay, DSM/IRFU/SAp }\end{array}$ \\
\hline Standard Key Words: & $\begin{array}{l}\text { Application specific integrated circuits, Astrophysics instrumentation, CdTe, } \\
\text { CdTe characterization, CdTe detectors, Charge sharing, Energy resolution, } \\
\text { Front-end electronics, Gamma-ray cameras, Gamma-ray spectroscopy, } \\
\text { Instrumentation, Semiconductor detectors, X-ray instrumentation, X-ray } \\
\text { detectors, X-ray spectroscopy }\end{array}$ \\
\hline
\end{tabular}




\title{
Low Energy characterization of Caliste HD, a fine pitch CdTe-based Imaging Spectrometer
}

\author{
Sébastien Dubos*, Olivier Limousin, Claire Blondel, Rémi Chipaux, Youri Dolgorouky, Olivier Gevin, \\ Yves Ménesguen, Aline Meuris, Thierry Orduna, Thierry Tourette and Aymeric Sauvageon.
}

\begin{abstract}
Caliste HD is a recently developed micro-camera designed for $X$ and gamma-ray astronomy, based on a $1 \times 1 \mathrm{~cm}^{2}$ CdTe Schottky pixelated detector. Its entire surface is composed of 256 pixels, disposed on a $16 \times 16$ pixel matrix. This spectrometer is buttable on its 4 sides and can be used to create a large focal plane. Caliste HD is designed for space environment. Its IDeF-X front-end electronics has a low power consumption, excellent noise performance and a wide dynamic range, from $2 \mathrm{keV}$ to $1 \mathrm{MeV}$. Moreover, electronic noise performances of this device were optimized to set the low level-energy threshold lower than 2 keV.
\end{abstract}

This paper focuses on the Caliste HD performance near the low energy limit. For this purpose, we have exposed the Caliste HD module to a mono-energetic $X$-ray beam, and set energies between 2 and $12 \mathrm{keV}$. We measured accurately the detection efficiency in this energy range and found it to be ranging from $39 \%$ to $75 \%$ for energies from $2.2 \mathrm{keV}$ to $11.6 \mathrm{keV}$, considering only particles detected in the single-event photopeak and ignoring events impinging between two adjacent pixels. This efficiency detection profile thereby highlights crucial effects of the Pt electrode opacity on Caliste HD low energy response, and suggests the presence of absorption zones at the interface between CdTe crystal and platinum. Respective thickness of each layer were estimated by simulation and confirmed by RBS (Rutherford Backscattering Spectroscopy).

Besides, using a mono-energetic beam allows fine energy resolution measurement, which was found to be ranging from 560 to $760 \mathrm{eV}$ FWHM between 2 and $12 \mathrm{keV}$. In addition, the linearity of this spectrometer and the issue of charge sharing between adjacent pixels were studied. This study revealed that spectroscopic performances remain excellent for such boundary operating conditions.

\section{INTRODUCTION}

$T_{\mathrm{m}}^{\mathrm{H}} \mathrm{cit}$ HE Caliste HD (HD is standing for "High Dynamic") module is the third generation of a development series of CdTe-based imaging spectrometers at CEA Saclay [1-3]. This advanced device is designed for future $\mathrm{X}$ or gamma-ray astronomy missions and Solar physics [4-9]. It operates

Manuscript received April 22, 2013.

*Corresponding author. Telephone: +33 (0) 169086489.

E-mail: sebastien.dubos@cea.fr

S. Dubos, O. Limousin, C. Blondel, A. Meuris, T. Orduna, T. Tourette and A. Sauvageon are with CEA Saclay, DSM/IRFU/SAp (Astrophysics Division), 91191 Gif-sur-Yvette Cedex, France.

R. Chipaux and O. Gevin are with CEA Saclay, DSM/IRFU/SEDI (Electronics, Detectors and Computer Science Division), 91191 Gif-surYvette Cedex, France.

Y. Dolgorouky is with APC Laboratory, 10 rue Alice Domon et Léonie Duquet, 75205 Paris Cedex 13, France.

Y. Ménesguen is with CEA Saclay, DRT/LIST/LNHB, 91191 Gif-surYvette Cedex, France. typically in the energy range between $2 \mathrm{keV}$ and $1 \mathrm{MeV}$, with excellent spatial resolution and spectroscopic performances.

The modular design of this small imaging-spectrometer which covers a $1 \mathrm{~cm}^{2}$ active surface equipped with 256 pixels, can be used to form large detection areas, composed of sets of modules arranged side by side and disposed at the focal plane of a space telescope to cover a large field of view, typically tens of arcmin for X-ray astronomy [10]. In addition, the small pitch of the pixelated detector of 625 micrometers provides an appropriate spatial resolution required for this kind of instrument.

As well as good spatial resolution is required, spectral performance is a crucial parameter for $\mathrm{X}$ and gamma-ray astronomy. Previous generations of imaging-spectrometers developed at CEA - named Caliste 64 [1] and Caliste 256 [2], with respectively 64 pixels of $1 \mathrm{~mm}$ pitch and 256 pixels of $580 \mu \mathrm{m}$ pitch - already showed excellent spectral performances and a low energy threshold estimated around $2 \mathrm{keV}$. New improvements on the Caliste HD module results in even better energy resolutions and the low energy threshold can be set below $2 \mathrm{keV}$ [3].

Such a low energy threshold is a major improvement for this kind of detector, as it offers additional information for observations that require multiple wavelengths: it can be useful in astrophysics to observe thermal and non-thermal phenomena in a unique observation. Also, having a unique detector for both low and high energies is interesting to avoid cross-calibration issues and minimize systematic errors in reconstructed spectra.

Significant detection efficiency is therefore required, as well as high energy resolution, energy response linearity and fine pixel pitch.

A detailed study of the detector behavior at these boundary conditions was performed. In particular, a fine measurement of detection efficiency was done using a flux-calibrated X-ray source for low energies, and particularly in the 2 to $6 \mathrm{keV}$ range. This energy band is interesting for the study of this type of CdTe imaging-spectrometer, as the photon collection efficiency that is supposed to be almost total for high energies, may sharply decrease below $6 \mathrm{keV}$. This behavior is suspected of being related to the absorption of the platinum entrance window, but some other effects can occur.

At first we present an overview of Caliste HD (Part II) and our experimental setup (Part III). Experimental results like integral non-linearity, imaging capabilities and energy resolution are considered. Later on, the data analysis process is detailed (Part IV). Then, we focus on results obtained for detection efficiencies at low energies (Part V). Finally, results 
obtained are discussed and extracted parameters compared to RBS measurements. We also consider issues related to charge sharing between pixels (Part VI).

\section{CAListe HD OVERVIEW}

Caliste HD [3] device can be divided into 3 parts: a pixelated Schottky CdTe detector subdivided into 256 pixels (first part) which is connected to a specially-developed frontend electronics, including 8 mixed analog-digital IDeF-X HD $[11,12]$ ASICs (Application Specified Integrated Circuit) each with 32 spectroscopic channels and disposed perpendicular to the detection surface (second part). This electrical body can communicate through a $4 \times 4$ pin grid array located at the opposite side of the detection surface (third part). The details of the assembly of the camera are presented Fig. 1.

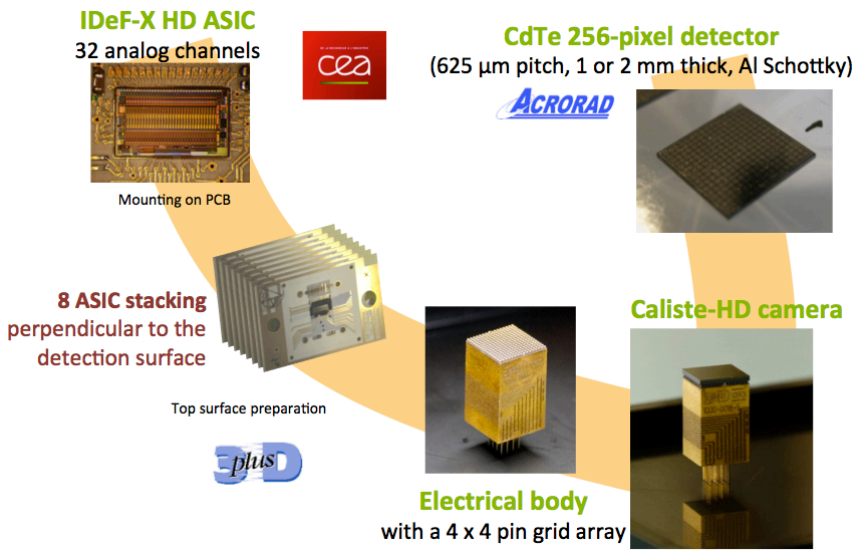

Fig. 1. Detailed components of a complete Caliste HD module.

The IDeF-X HD is entirely designed at CEA Saclay (France) while the CdTe crystal is manufactured by Acrorad (Japan). The integration of these sub-parts into a detection module is realized by 3D Plus (France).

The CdTe detector is surrounded by a $1 \times 1 \mathrm{~cm}^{2} \mathrm{Al}$ anode segmented into an array of $16 \times 16$ pixels and a Pt cathode. The latter constitutes the entrance window for incoming photons. The total thickness of the crystal in this work was set to $1 \mathrm{~mm}$.

As shown on Fig. 2, each pixel is $525 \mu \mathrm{m}$ large, the gap between two adjacent pixels is $100 \mu \mathrm{m}$ and the matrix is surrounded by a $20-\mu \mathrm{m}$ width guard ring, also separated from the pixels by a gap of $100 \mu \mathrm{m}$. The detection surface is $98 \mathrm{~mm}^{2}$, subtracting the area of the guard ring and its $100 \mu \mathrm{m}$ gap to the outermost pixels. This detection surface is composed of a full metallized pixel surface of $70.56 \mathrm{~mm}^{2}$ (72\% of the total) and a metal free inter-pixel zone of $27.45 \mathrm{~mm}^{2}$ (28\% of the total). As a consequence, for a homogeneous impinging photon flux, $28 \%$ of photons are likely to hit an inter-pixel surface. This ratio can be compared to the charge-sharing ratio obtained by prediction and experimentally (Part VI).

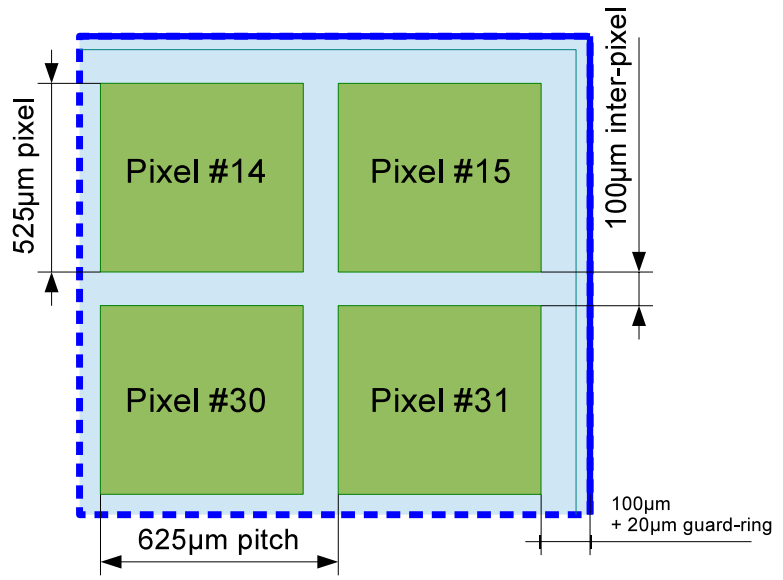

Fig. 2. Scheme of the right top corner of the subdivided anode with pixel size and inter-pixel zone, plus the $20 \mu \mathrm{m}$ guard ring.

Each pixel is connected to a spectroscopic channel of an IDeF-X HD ASIC. A channel includes charge pre-amplifier, shaper, baseline holder and peak detector as well as analog memory. A large set of programmable parameters is available on the chip to optimize the signal to noise ratio such as peaking time, threshold or gain value. In addition, low level thresholds can be set individually in each channel.

The module readout is very flexible and the user can arbitrarily decide to use single or multiple events allowing charge sharing reconstruction or particle ionizing track rejection for instance.

Caliste HD is designed for space applications and complies with corresponding fabrication standards as well as radiation hardness, reliability and low power properties.

\section{EXPERIMENTAL SETUP}

In order to characterize Caliste HD near the low energy threshold, we need a calibrated low energy X-ray beam. This requirement is satisfied by the use of SOLEX (Source Of LowEnergy X-rays) [13], a facility located at CEA Saclay. In addition, a dedicated setup was developed to realize the interface (mechanical, vacuum, electrical and alignments) between SOLEX and Caliste HD.

\section{A. SOLEX facility}

The SOLEX facility is designed to produce a monoenergetic X-ray beam from 1 to $20 \mathrm{keV}$.

It is composed of an X-ray tube, which produces a polychromatic beam. We can make fine energy selection by directing the beam through a monochromator crystal. Indeed, by changing the incident angle of the beam on the monochromator, we can select a specific wavelength (Bragg law). Different monochromators can be used, depending on desired energies (Table I).

For our experiments, InSb was chosen for characterization runs between 2 and $5.5 \mathrm{keV}$ while a quartz crystal was used at higher energies up to $15 \mathrm{keV}$ 
TABLE I. EXAMPLES OF MONOCHROMATORS AND ASSOCIED ENERGY RANGES

\begin{tabular}{lll} 
Crystal & Minimal energy $(\mathrm{eV})$ & Maximal energy $(\mathrm{eV})$ \\
\hline InSb (111) & 1964 & 6809 \\
Quartz (R) & 4086 & 14957 \\
\hline
\end{tabular}

The mono-energetic beam is directed to the Caliste characterization setup through an optional and adjustable collimation system to obtain the full spot shape on the detector surface.

Making an absolute efficiency measurement requires that the complete mono-chromator system can rotate and guide the beam to a calibrated proportional gas counter, which gives incident flux reference values [14].

The beam flux is adjustable between 100 and 1000 counts/s, for a typical acquisition time comprised between 10 and 15 minutes per run.

\section{B. Caliste HD characterization setup}

The Caliste characterization chamber is disposed at the output of the SOLEX beam.

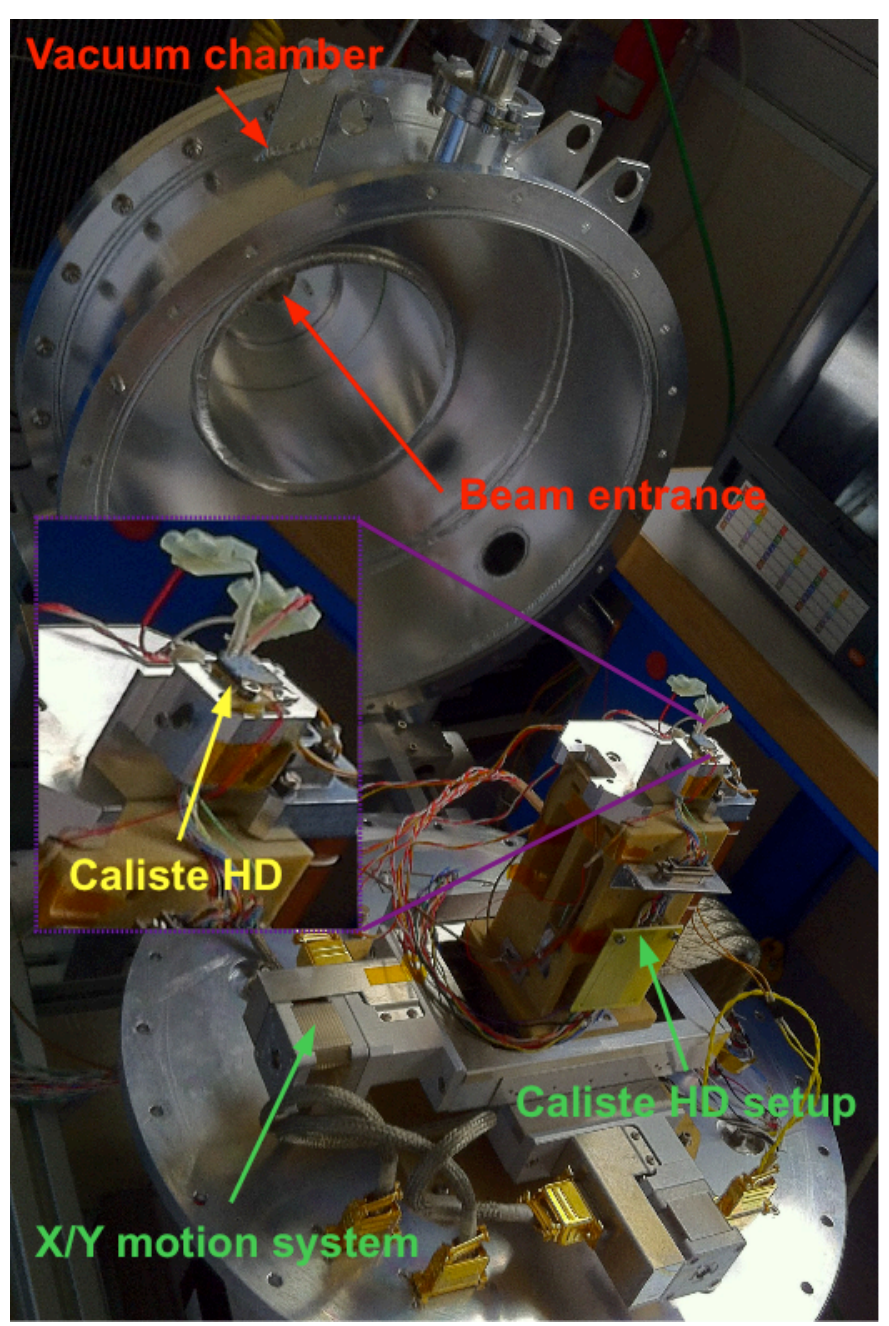

Fig. 3. Caliste HD characterization setup (opened).

A bias voltage of $310 \mathrm{~V}$ was applied to the detector, and the peaking time set to $10.7 \mu \mathrm{s}$. These parameters provide optimal conditions for low energy measurements. A vacuum equal to
$10^{-5}$ mbar was established in the vessel, and an active thermal control maintains the sensor at $-10^{\circ} \mathrm{C}$. Caliste HD has its own internal set of thermal sensor which are used as house-keeping to monitor the operating temperature time to time.

Additionally, a 2-axis motion system is specially developed to allow centering the detector into the beam.

Communication with Caliste HD is established through a FPGA connected to the module. By the same way, we read out the signal amplitudes and time-tags corresponding to triggered pixels. The telemetry obtained is transferred to a computer by a spacewire link, acquired, stored and converted into the standardized FITS file format for off-line analysis. A real-time quick look facilitates alignments and proper Caliste HD operations.

\section{IMAGING AND SPECTROSCOPIC PROPERTIES}

Two series of runs were performed: 11 runs between 2.1 and $3.1 \mathrm{keV}$ and 39 runs between 3 and $12 \mathrm{keV}$, for a total of 50 runs. We emphasized the $2-6 \mathrm{keV}$ band with 40 runs in this energy range. Some additional points up to $16 \mathrm{keV}$ were also acquired for energy calibration, including linearity check for the entire energy range of interest.

The ability of Caliste HD to detect and image the beam shape, the response linearity at low energies, gain distribution and energy resolution values below $12 \mathrm{keV}$ were studied.

\section{A. Energy calibration \& linearity}

At first, it was necessary to make an energy calibration of each individual pixel of Caliste HD for further analysis. Moreover, the linearity of this spectrometer for the lowest energies has to be clearly established.

For each pixel, the amplitude (in ADU - Analog/Digital Units) of the photopeak was plotted for all beam energies, and fitted by a linear function (Fig. 4). Calibration parameters obtained for each pixel were stored into a calibration file, systematically used in the following.

The mean value of the gain obtained is $68 \mathrm{ADU} / \mathrm{keV}$, or $1895 \mathrm{ADU} / \mathrm{fC}$.

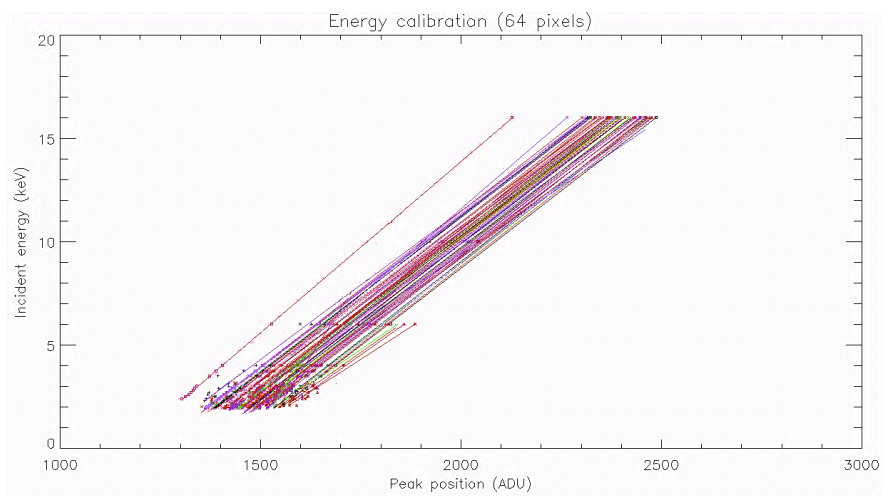

Fig. 4. Incident energy (keV) as a function of peak amplitude (ADU), for the 64 most active pixels - First campaign.

The INL (Integral Non-Linearity) is found to be $0.63 \%$ in the $2-6 \mathrm{keV}$ range. The linearity of Caliste HD is therefore 
demonstrated here. Furthermore, the gain standard deviation for the 64 most active pixels during the runs is lower than $5 \%$, which shows a good uniformity of the pixel matrix gain.

\section{B. Imaging capabilities}

The $16 \times 16$ fine pitch pixel array of Caliste HD was used to record the beam shape and to align the entire spot on the detector surface.

For almost all runs, the spot size was constrained to approximately $3 \times 2 \mathrm{~mm}^{2}$. An example of image obtained for the lowest energy $(2.137 \mathrm{keV})$ is displayed on Fig. 5. Due to the effect of the collimator used to reduce the beam shape, we obtained a non-symmetric beam on Caliste HD. Considering the X-axis for instance; a smooth increase of the count rate on the left side and a sharp cut on the right side are visible.

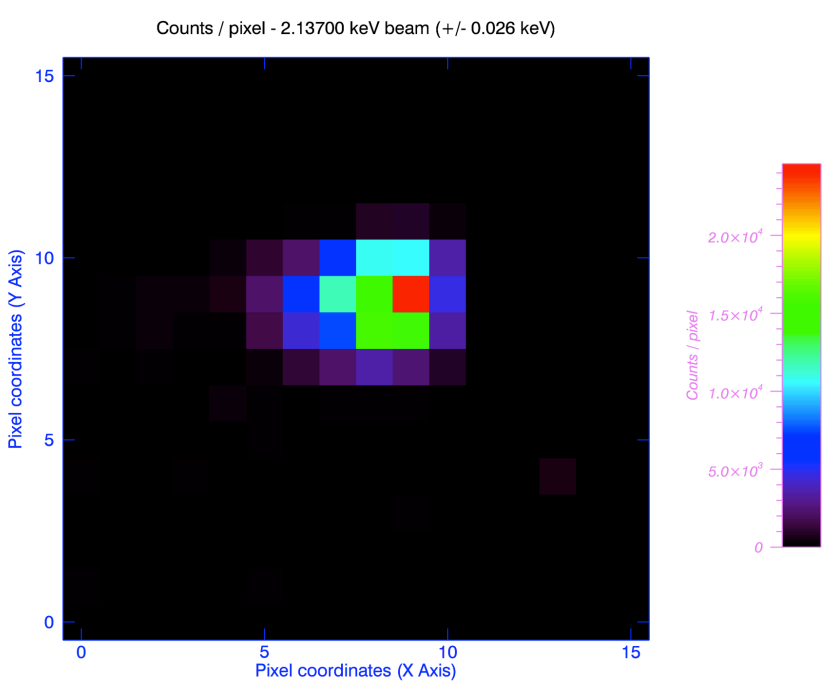

Fig. 5. Image obtained with a $2.137 \mathrm{keV}$ spot. With a pitch of 625 micrometers, we can estimate the spot size to a maximum of $3.75 \mathrm{~mm}$ (X axis) and $2.5 \mathrm{~mm}$ ( $\mathrm{Y}$ axis). The color scale represents the count rate of each pixel.

\section{Data Analysis protocol}

Using our calibration file, we can reconstruct the sum spectrum, by summing all individual spectra obtained for each pixel. An example of sum spectrum obtained at $2.14 \mathrm{keV}$ is shown on Fig. 6. Sum spectra are built using single events (1 hit pixel at a time) and multiple events $(2,3$ or 4 simultaneously hit pixels, due to charge-sharing between neighboring pixels). According to the count rate at most equal to 1000 counts/s for the entire detector and the peaking time settled to $10.7 \mu \mathrm{s}$, it is very unlikely that spurious double events occur.

On the sum spectrum presented on Fig. 6, the low-level energy threshold is estimated to be as low as $1.3 \mathrm{keV}$. Due to the fact that this threshold is set close to the beam energy and regardless the energy distribution between neighbor pixels, almost no photon can be detected as a shared event with a $2.137 \mathrm{keV}$ source.
With the increasing beam energy, the difference between impinging photons and low threshold becomes more significant to allow the detection of split events. These additional counts, resulting of charge-sharing between 2,3 or 4 neighbor pixels degrade the energy resolution of the sum spectrum. Moreover, these reconstructed spectra show a significant energy loss (cf. Part VI), which implies that the beam energy cannot be accurately determined without an appropriate correction.

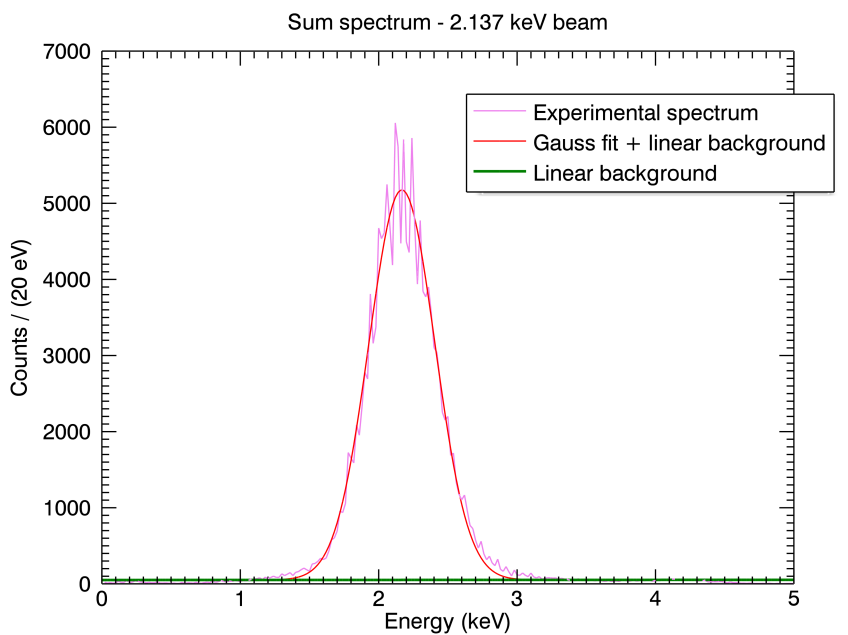

Fig. 6. Example of sum-spectrum obtained with the $2.137 \mathrm{keV}$ beam. The photopeak can be fitted by a Gaussian function.

By considering only spectra generated with single events between 2 and $12 \mathrm{keV}$, we can thereby suppress the contribution of shared events and consider a constant effective detection surface, for simplification. It is very useful for instance, to quantify the effects of the Pt entrance electrode and other opaque layers on the detection efficiency profile without other additional effects.

For all runs, a Gaussian function is fitted to the right side of the photopeak produced with single events. Derived parameters are used to determine the integral of the peak and energy resolution. On the other hand, a dead-time correction is applied to compute the count rate. The latter is compared to the incoming flux for detection efficiency extraction in the single event photopeak. Proceeding this way, we found the efficiency to be $42.71 \pm 0.94 \%$ at $2.137 \mathrm{keV}$.

Fitting the right side of the photopeak allows us to filter the contribution of partial events produced by asymmetric charge sharing where a fraction of the charge remains below the threshold. Such events are triggering one channel only and are consequently considered as singles, creating a peak tail towards the low energy.

Conversely, to derive the total detection efficiency, the entire sum spectrum was integrated and compared with the incoming photon flux at a given energy, after dead-time correction. 


\section{Energy resolution}

Energy resolutions (FWHM) were derived for our different series of runs. Values were found between 560 and $758 \mathrm{eV}$ in the studied energy range, considering the sum spectrum of all pixels, or between 441 and $655 \mathrm{eV}$ if we consider only one pixel centered on the beam. We observe a slight degradation of the resolution between 2.1 and $2.5 \mathrm{keV}$, but no clear evolution is visible at higher energies (Table II).

TABLE II. EXAMPLES OF ENERGY RESOLUTIONS FOR DIFFERENT BEAM ENERGIES

\begin{tabular}{lll} 
Beam energy & FWHM (1 single pixel) & FWHM (all pixels) \\
\hline $2.137 \mathrm{keV}$ & $441 \mathrm{eV}$ & $560 \mathrm{eV}$ \\
$2.172 \mathrm{keV}$ & $512 \mathrm{eV}$ & $579 \mathrm{eV}$ \\
$2.272 \mathrm{keV}$ & $596 \mathrm{eV}$ & $626 \mathrm{eV}$ \\
$2.372 \mathrm{keV}$ & $638 \mathrm{eV}$ & $669 \mathrm{eV}$ \\
$2.472 \mathrm{keV}$ & $637 \mathrm{eV}$ & $710 \mathrm{eV}$ \\
$2.5-16 \mathrm{keV}$ & $604-655 \mathrm{eV}$ & $630-758 \mathrm{eV}$ \\
\hline
\end{tabular}

These results are close, and better compared to energy resolution measurements performed on other Caliste HD samples, where we found $820 \mathrm{eV} \mathrm{FWHM}$ at $14 \mathrm{keV}$ on a sum spectrum of a ${ }^{241} \mathrm{Am}$ source $[3,11]$.

\section{DETECTION EFFICIENCY}

Experimental values of detection efficiencies obtained by considering single and multiple events for beam energies between 2 and $12 \mathrm{keV}$ (50 runs) are displayed Fig. 7. We emphasized the energy range between 2 and $6 \mathrm{keV}$ (40 runs) on Fig. 8.

Data analysis for each run is similar, and different measurement series are presented: the first campaign with the InSb monochromator used in SOLEX facility, and the second campaign with both InSb and quartz crystals.

Considering single events, uncertainties on detection efficiencies remain below $1 \%$ for a majority of runs from the first campaign. They are approximately between 2 and $4 \%$ for the second campaign due to less favorable operating conditions. Uncertainties on total detection efficiencies are below $1 \%$ for almost all runs.

Determined values for detection efficiency considering single events are all comprised between $39.49 \pm 0.81 \%$ (2.172 keV beam) and 74.23 $\pm 1.86 \%$ (11.525 keV beam).

Regarding all events, total detection efficiency values are between $40.43 \pm 0.38 \%(2.172 \mathrm{keV})$ and $98.82 \pm 1.54 \%$ $(12 \mathrm{keV})$. For the lowest energy, the detection efficiency measured for single events is also the total detection efficiency of the entire detector since no split event could trigger Caliste HD in these conditions.

X-ray absorption edges of platinum $\left(\mathrm{M}_{\mathrm{IV}}\right.$ edge at $2.204 \mathrm{keV}$, $\mathrm{M}_{\mathrm{III}}$ edge at $2.649 \mathrm{keV}$ and eventually $\mathrm{L}_{\mathrm{III}}$ edge at $11.568 \mathrm{keV}$ ) and also tellurium absorption edges $\left(\mathrm{L}_{\mathrm{III}}\right.$ edge at $4.345 \mathrm{keV}, \mathrm{L}_{\mathrm{II}}$ edge at $4.616 \mathrm{keV}$ ) appear on our efficiency curves and particularly on the efficiency curve measured for single events, as explained Part. IV-C.
We can also detect an effect of cadmium absorption edges $\left(\mathrm{L}_{\mathrm{III}}(\mathrm{Cd})=3.554 \mathrm{keV}, \mathrm{L}_{\mathrm{II}}(\mathrm{Cd})=3.742 \mathrm{keV}\right)$, but less visible considering error bars.

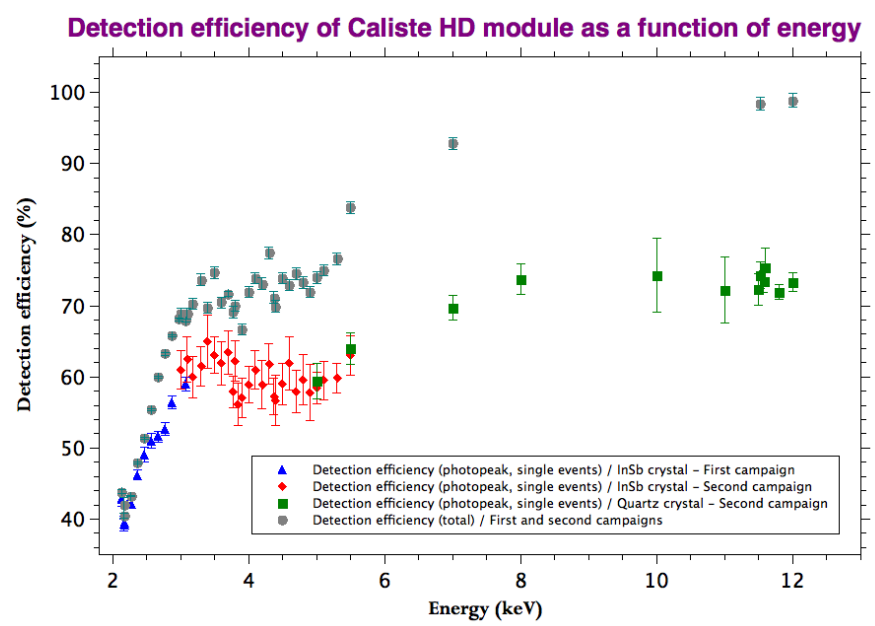

Fig. 7. Detection efficiency measured for single and multiple events, between 2.137 and $12 \mathrm{keV}$. Two series of runs were represented, for different monochromators used in SOLEX facility.

Variations of experimental detection efficiency for these characteristic energies imply an effect of the $\mathrm{Pt}$ entrance electrode, as well as the presence of a CdTe-like thin dead layer that absorb a significant fraction of low-energy photons. Attenuation due to these elements progressively decreases as the beam energy increases.

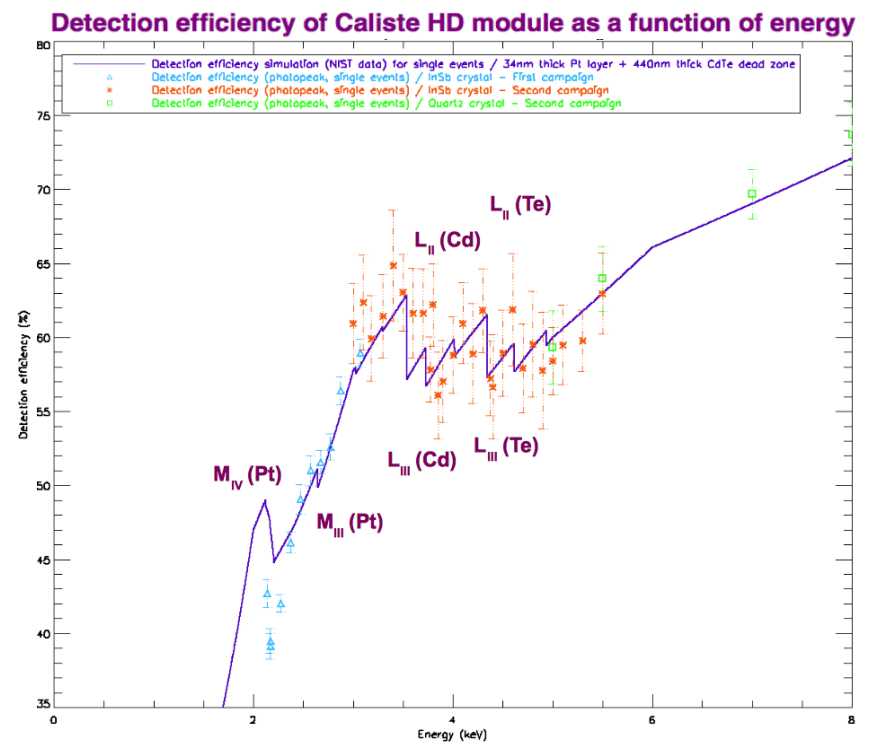

Fig. 8. Detection efficiency measured and simulated for single events, between 2.137 and $8 \mathrm{keV}$. Two series of runs were represented, for different monochromators used in SOLEX facility. Characteristic X-rays absorption edges are marked.

At higher energies, the efficiency curve saturates after $8 \mathrm{keV}$, with values that tend toward $75 \%$ for single events. Taking into account the total efficiency, including multiple events, the curve saturates at $98 \%$. 
On Fig. 7 and 8, we can identify absorption edges of Pt, $\mathrm{Cd}$ and Te. These lines are useful to identify the nature of different absorption layers.

\section{DISCUSSION}

\section{A. Charge-sharing effects}

Experimental detection photopeak efficiencies for single events only are presented in Fig. 7 and 8. This unusual presentation of singles is to facilitate the analysis of the entrance window absorption ignoring charge sharing and split event effects that would corrupt dead layer properties extraction.

Assuming a uniform illumination of the sensor entrance face, $72 \%$ of the photons interact to the vertical of a single pixel while $28 \%$ hit the inter-pixel gap, most likely causing a multiple event if the energy deposit is high enough. Actually, deriving this ratio of single event will lead to a slightly higher value due to the distortion of electric field lines near the pixels which gives the pixel a effective area larger than the metal surface. This effective area of a pixel is getting closer and closer to the physical pixel surface as the applied bias voltage gets higher.

Iniewski et al. [15] proposed a geometric-based model to estimate the probability of shared events in CdTe pixelated detectors. Meuris et al. [16] applied this model to the Caliste 64 geometry. This method describes very well chargesharing rates observed for different operating conditions, including voltage and detector geometries.

Considering $V$ the applied bias voltage, $d$ the thickness of the detector, $k$ the Boltzmann's constant and $T$ the temperature, the characteristic size of the electron cloud formed at the anode is, according to the diffusion equation and the Einstein's relationship:

$$
\sigma=d \sqrt{\frac{2 k T}{q V}}
$$

Applying the model to our operating conditions $\left(-10^{\circ} \mathrm{C}\right.$, $310 \mathrm{~V}$ bias voltage, $1 \mathrm{~mm}$ thick detector) one find a charge cloud spreading of approximately $12 \mu \mathrm{m}$ when the cloud goes across the entire thickness, which is reasonable at such low energies.

On the other hand, Equation (2) gives the corresponding probability of a multiple event according to the geometry:

$P=1-\left(\frac{a+2 c-2 r}{a+g}\right)^{2}$

Where $a$ is the pixel size $(525 \mu \mathrm{m}), g$ the inter-pixel gap $(100 \mu \mathrm{m}), c$ the collection width $(\min (g / 2, a / 20)=a / 20$ here $)$ and $r$ the radius of the electron cloud $(1.15 \sigma)$.

We find $P=23 \%$ (which can be compared to the geometric ratio of $28 \%$ probability to hit an inter-pixel surface).

According to this model, single events represents $77 \%$ of the incoming flux, regardless the energy. The effective ratio of shared events depends however on the value of the low energy threshold; if we consider double events for instance, in some cases the energy distribution between the two adjacent pixels is unbalanced and one of the two induced signals is below the threshold, and this event is marked as a single one.

This effect is even more pronounced as the beam energy is near the threshold. To overcome it and to have more representative values on the real charge-sharing ratio, we considered a whole ${ }^{241} \mathrm{Am}$ spectrum measured with comparable operating conditions by Caliste HD. The chargesharing rate was $23.5 \%$, with $76.5 \%$ single events. These results nicely match the model.

For low energy measurements, when the threshold is not negligible compared to the deposited energy, the chargesharing rate effectively measured can be very different. On Table III, some experimental charge-sharing rates are displayed.

TABLE III. EXAMPLES OF CHARGE-SHARING RATES MEASURED FOR DIFFERENT BEAM ENERGIES

\begin{tabular}{lll} 
Beam energy & Single-events rate & Multiple-events rate \\
\hline $2.137 \mathrm{keV}$ & $99.33 \%$ & $0.67 \%$ \\
$2.172 \mathrm{keV}$ & $99.25 \%$ & $0.75 \%$ \\
$2.272 \mathrm{keV}$ & $99.46 \%$ & $0.54 \%$ \\
$3.072 \mathrm{keV}$ & $99.03 \%$ & $0.97 \%$ \\
$3.672 \mathrm{keV}$ & $98.17 \%$ & $1.83 \%$ \\
$6 \mathrm{keV}$ & $93.93 \%$ & $6.07 \%$ \\
$7 \mathrm{keV}$ & $93.40 \%$ & $6.60 \%$ \\
$10 \mathrm{keV}$ & $89.11 \%$ & $10.09 \%$ \\
$12 \mathrm{keV}$ & $83.57 \%$ & $16.43 \%$ \\
\hline
\end{tabular}

Detected charge-sharing rate increases when energy is up to $3.1-3.2 \mathrm{keV}$ : below, almost all shared events are chance triggers.

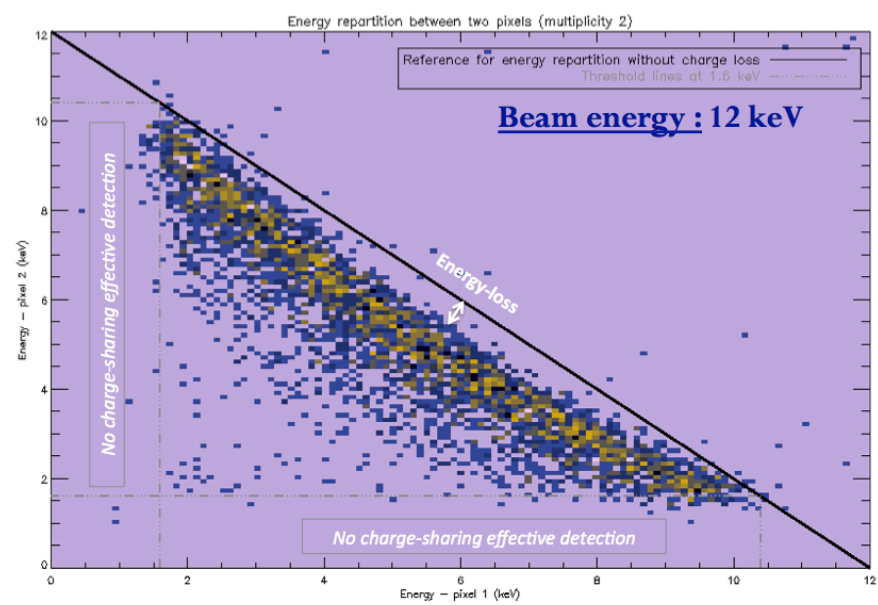

Fig. 9. Energy repartition between two neighbor pixels touched simultaneously, with a $12 \mathrm{keV}$ beam.

By increasing beam energies, these values tend to higher charge-sharing ratio as predicted before. Fig. 9 represents the correlation plot obtained considering double events for a $12 \mathrm{keV}$ beam. We clearly see the charge-sharing profile of double events for the beam, plotting a pixel response against any neighbor pixel for split events. The repartition of shared events seems to be homogeneous along permitted energies. 
Extrapolating the amount of shared events detected in the permitted range, we estimate a charge-sharing probability of $22.40 \%$. This extrapolation allows computing the expected charge-sharing fraction for a virtually zeroed threshold level. This value is gain matching the expected split event rate.

In the following section, we keep this value of $22.7 \%$ for charge-sharing probability.

Moreover, we symmetrically see on Fig. 9 an energy loss for split double events: reconstructed spectrum gives $10 \%$ charge loss for the peak value (Fig. 10). This energy loss is relatively high compared to Caliste 64 [16] $(1.2 \mathrm{keV}$ at $12 \mathrm{keV}$ here, and $0.3 \mathrm{keV}$ at $60 \mathrm{keV}$ for Caliste 64).

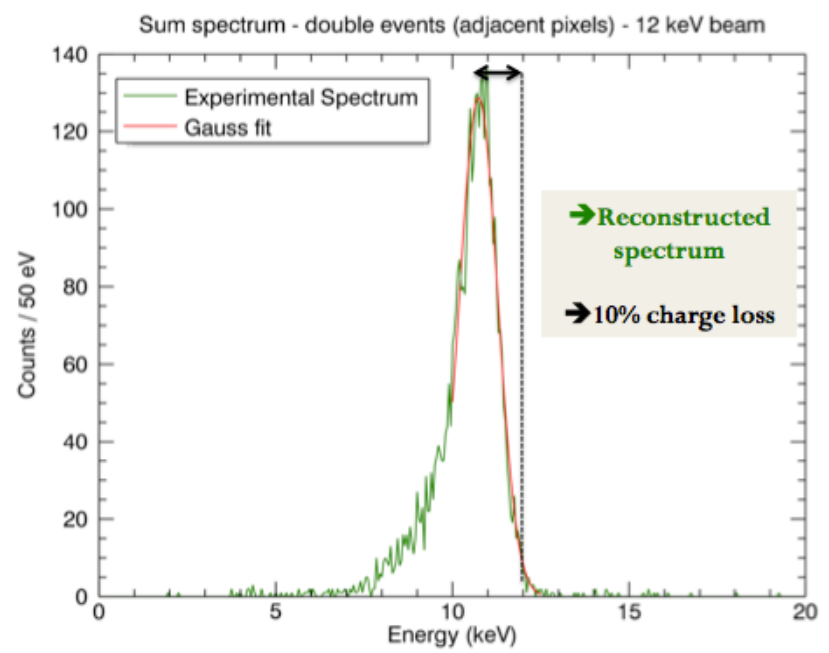

Fig. 10. Reconstructed spectrum for double events, with a $12 \mathrm{keV}$ beam.

\section{B. Detection efficiency simulation}

As explained in section $\mathrm{V}, \mathrm{X}$-ray absorption edges are visible on the efficiency curve for $\mathrm{Pt}$, Te and $\mathrm{Cd}$ elements. As the presence of a platinum layer that covers the entire entrance electrode, is already established, its exact thickness has to be determined accurately. Also, the characteristics of a dead layer composed of $\mathrm{Cd}$ and $\mathrm{Te}$ can be estimated.

It is possible to find an approximated value for the CdTe dead layer thickness by considering the $\mathrm{L}_{\mathrm{III}}(\mathrm{Te})$ edge at $4.345 \mathrm{keV}$. We measured an efficiency of $67.18 \pm 2.84 \%$ for $4.300 \mathrm{keV}$, and $62.21 \pm 2.52 \%$ for $4.375 \mathrm{keV}$. It implies $7.98 \pm$ $0.45 \%$ efficiency loss at this edge.

Equation 3 gives an estimation of the CdTe dead layer thickness $x$, for $A_{\text {edge }}=$ Efficiency before edge $/$ Efficiency after edge $=$ 1.0798:

$$
x=\frac{\ln \left(A_{\text {edge }}\right)}{\left(\mu_{\text {after edge }}-\mu_{\text {before edge }}\right) \times \varrho}
$$

Where $\mu$ and $\rho$ are the CdTe mass attenuation coefficients and density respectively. Applying to CdTe, we find a thickness of the CdTe dead layer equal to $475 \pm 28 \mathrm{~nm}$.

The experimental profile obtained Fig. 8 was fitted using NIST data for mass attenuation coefficients for Pt and CdTe [17]. A $1 \mathrm{~mm}$ thick CdTe detector with adjustable sensitive volume and dead zone disposed under a Platinum-made entrance window was simulated. We also considered a fraction of $77.34 \%$ single events.

The best fit was obtained by choosing a $34 \mathrm{~nm}$ thick $\mathrm{Pt}$ layer and a $440 \mathrm{~nm}$ thick CdTe dead layer (Fig. 8). With this simulation, only 8 experimental points out of 40 in the 2$8 \mathrm{keV}$ range are not well fitted. The CdTe-like dead layer was slightly lower compared to predicted values. Iwase et al. [18] also identified a thin $\mathrm{TeO}_{\mathrm{x}}$ layer on CdTe crystals.

The model fitting is not accurate and systematically over estimated at very low energy, close to the threshold. We assume that this effect is due to the dispersion of the effective low threshold values from pixel to pixel, which results in a deficit on the count rate - as a matter of fact; energy calibration reveals a smooth standard deviation on the gain and offset values $(2.11 \%$ and $2.71 \%$ respectively).

\section{Experimental verification of layers thickness}

We used an additional technique to explore respective thicknesses and composition of the different material layers in the detector that have been derived from efficiency curve fitting. A representative CdTe sample was analyzed by RBS (Rutherford Backscattering Spectroscopy) at the ARAMIS facility, CSNSM, Orsay, France.

ARAMIS is a Tandem-type accelerator working with a minimal high-voltage of $2 \mathrm{MV}$. It delivers $\mathrm{He}^{2+}$ beam when used in Van de Graaf mode [19]. This beam is used to probe superficial layers analyzing the backscattered spectrum.

A CdTe detector, disposed into a vacuum chamber, was exposed to a monoenergetic flux of $3.2 \mathrm{MeV} \mathrm{He}^{2+}$ ions. Energies of backscattered ions were measured with a Ge spectrometer, for a scattering angle of $165^{\circ}$.

From the energy spectrum obtained in Fig. 11 two main structures are visible: single peaks which represent the energy lost in scattered events with sample nuclei (depending on the sample atomic numbers) and a large continuum for low energies which depends on the stopping-power of the electrons in the sample (depending on the electron density and distances traversed across the sample material). Due to this second effect, energy peaks are progressively shifted towards lower energies.

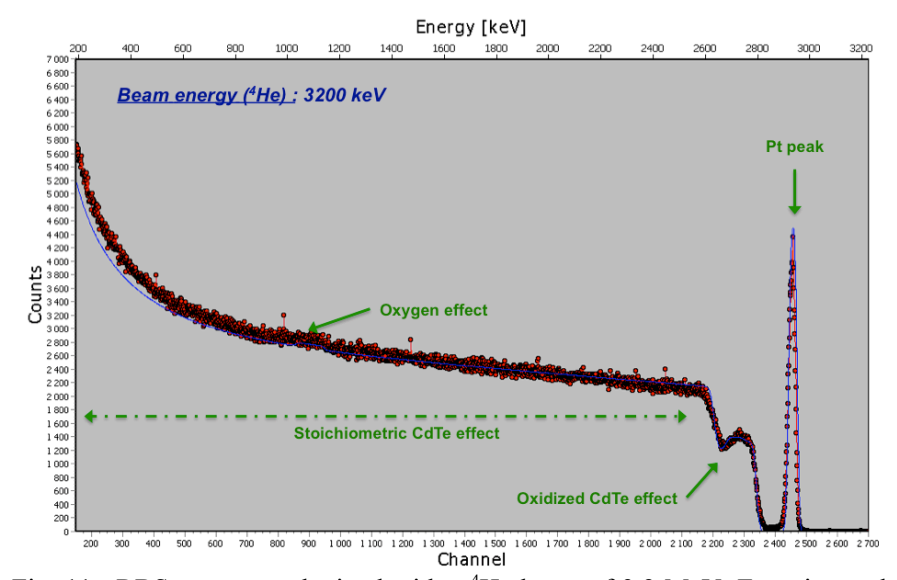

Fig. 11. RBS spectrum obtained with a ${ }^{4} \mathrm{He}$ beam of $3.2 \mathrm{MeV}$. Experimental points are in red, and the simulated curve is blue. 
Analyzing the resulting RBS spectrum, respective thickness and composition of individual layers are estimated using peaks shifts and width as well as relative peak heights.

We used the SIMNRA software [20] to fit this spectrum. We constrained the fit with three successive layers:

o a Pt layer with an adjustable thickness,

o another adjustable layer composed with an adjustable ratio of $\mathrm{Cd}$, $\mathrm{Te}$ and $\mathrm{O}$,

o a stoichiometric CdTe layer with an infinite thickness.

The best fit was obtained and over plotted in Fig. 9 with the following parameters:

- a platinum layer of $29 \mathrm{~nm}$,

- A $392 \mathrm{~nm}$ thick layer composed of cadmium (1.52\%), tellurium (30.35\%) and oxygen (68.13\%).

The layer thickness of the Pt electrode is very well matching the $34 \mathrm{~nm}$ thick Pt layer derived from efficiency curve fitting on a different sample, bur same production batch. The second layer is found to be a $\mathrm{TeO}_{2}$ oxidized dead layer comparable to the suspected $440 \mathrm{~nm}$ dead layer. RBS allows the identification of the dead layer nature.

\section{CONCLUSION}

In this paper, we described the characterization of the Caliste HD imaging-spectrometer at boundary conditions, as close as possible to the low energy threshold. With a threshold estimated to be $1.3 \mathrm{keV}$, a $2.1 \mathrm{keV}$ spot is easily detected and the detection efficiency is estimated equal to $43 \%$ for single events. This is a very useful information for application in space astrophysics where Caliste broad energy range is demonstrated. Moreover, precise measurement allows accurate sensitivity estimation of imaging spectrometers.

The linearity for low energies was also clearly verified, as well as energy resolution found between 560 and $760 \mathrm{eV}$.

Identification of successive X-ray absorption edges on the increasing efficiency curve allowed us to estimate the nature and the thickness of different absorption layers: the $\mathrm{Pt}$ entrance electrode and also a probable $\mathrm{TeO}_{2}$ dead zone. These layers limit the efficiency for the lowest energy ranges, typically below $8 \mathrm{keV}$. For higher energies, the detection efficiency rapidly increases and tends to very high values.

Respective thicknesses and compositions of superficial layers were also confirmed by RBS measurement made using the ARAMIS platform. We found a $29 \mathrm{~nm}$ thick Pt layer surrounding a $392 \mathrm{~nm}$ thick $\mathrm{TeO}_{2}$ dead layer.

By using SOLEX and ARAMIS, we acquired complete and accurate knowledge of Caliste HD at low energy down to $2 \mathrm{keV}$.

\section{ACKNOWLEDGMENTS}

We thank Cyril Bachelet (CSNSM, Orsay, France) who gave us the possibility to make RBS measurements on the ARAMIS facility. His help for data analysis was also precious.

This work is performed in a frame of a PhD Grant supported by CEA and CNES.

\section{REFERENCES}

[1] A. Meuris et al., "Caliste 64, an Innovative CdTe Hard X-Ray MicroCamera", IEEE Transactions on Nuclear Science, vol. 55, no. 2, pp. 778-784, April 2008.

[2] O. Limousin, et al., "A CdTe imaging spectrometer for space science with a $580 \mu \mathrm{m}$ pixel pitch", Nuclear Instruments and Methods in Physics Research Section A: Accelerators, Spectrometers, Detectors and Associated Equipment, Volume 647, Issue 1, 11 August 2011, Pages 4654.

[3] A. Meuris, et al., "Caliste HD: A new fine pitch $\mathrm{Cd}(\mathrm{Zn}) \mathrm{Te}$ imaging spectrometer from $2 \mathrm{keV}$ up to $1 \mathrm{MeV}$ ", 2011 IEEE Nuclear Science Symposium and Medical Imaging Conference (NSS/MIC), pp. 44854488, 23-29 Oct. 2011.

[4] T. Takahashi et al., "The ASTRO-H Mission", SPIE Proc. Conf., vol. 7732, pp. $77320 \mathrm{Z}$ (2010).

[5] F. A. Harrison et al., "The Nuclear Spectroscopic Telescope Array (NuSTAR)", SPIE Proc. Conf., vol. 7732, pp. 77320S (2010).

[6] G.Tagliaferri et al., "NHXM: a new hard X-ray imaging and polarimetric mission", SPIE Proc. Conf., vol. 7732, pp. 773217 (2010).

[7] P. Ferrando et al., "The COSPIX mission: focusing on the energetic and obscured Universe", $25^{\text {th }}$ Texas Symp. on Relativistic Astrophysics Proc., PoS(Texas 2010) 254 (2011).

[8] P. Ferrando et al., "Simbol-X: mission overview", SPIE Proc. Conf., vol. 6266, pp. 62660 (2006).

[9] A. Meuris, et al., "Caliste-SO X-ray micro-camera for the STIX instrument on-board Solar Orbiter space mission", Nuclear Instruments and Methods in Physics Research Section A: Accelerators, Spectrometers, Detectors and Associated Equipment, Volume 695, 11 December 2012, Pages 288-292.

[10] O. Limousin, "MACSI: an $8 \mathrm{~cm}^{2} \mathrm{CdTe}$ Detection Plane for TimeResolved Hard X-Ray Imaging with High Spectroscopic Capability", 2012 IEEE Nuclear Science Symposium and Medical Imaging Conference (NSS/MIC/RTSD), Talk \#R10-2, Oct. 29 2012-Nov. 32012.

[11] A. Michalowska et al., "IDeF-X HD: A low power multi-gain CMOS ASIC for the readout of $\mathrm{Cd}(\mathrm{Zn}) \mathrm{Te}$ detectors", 2010 IEEE Nuclear Science Symposium and Medical Imaging Conference (NSS/MIC/RTSD), pp. 1556-1559, Oct. 30 2010-Nov. 62010.

[12] O. Gevin et al., "Imaging X-ray detector front-end with high dynamic range: IDeF-X HD", Nuclear Instruments and Methods in Physics Research Section A: Accelerators, Spectrometers, Detectors and Associated Equipment, Volume 695, 11 December 2012, Pages 415-419.

[13] C. Bonnelle et al., "SOLEX: a tunable monochromatic X-ray source in the 1-20 keV energy range for metrology", Nuclear Instruments and Methods in Physics Research Section A: Accelerators, Spectrometers, Detectors and Associated Equipment, Volume 516, Issues 2-3, 11 January 2004, Pages 594-601.

[14] Y. Ménesguen, M.-C. Lépy, "Efficiency calibration and surface mapping of an energy-dispersive detector with SOLEX: A compact tunable monochromatic X-ray source"", Nuclear Instruments and Methods in Physics Research Section A: Accelerators, Spectrometers, Detectors and Associated Equipment, Volume 695, 11 December 2012, Pages 193-196.

[15] K. Iniewski et al., "Modeling charge-sharing effects in pixellated CZT detectors", Nuclear Science Symposium Conference Record, 2007. NSS '07. IEEE, vol.6, no., pp.4608-4611, Oct. 26 2007-Nov. 32007.

[16] A. Meuris, O. Limousin, C. Blondel, "Charge sharing in CdTe pixilated detectors", Nuclear Instruments and Methods in Physics Research Section A: Accelerators, Spectrometers, Detectors and Associated Equipment, Volume 610, Issue 1, 21 October 2009, Pages 294-297.

[17] http://physics.nist.gov/PhysRefData/XrayMassCoef/ComTab/telluride.ht $\mathrm{ml}$

[18] Y. Iwase, M. Funaki, A. Onozuka, M. Ohmori, "A 90 element CdTe array detector", Nuclear Instruments and Methods in Physics Research Section A: Accelerators, Spectrometers, Detectors and Associated Equipment, Volume 322, Issue 3, 15 November 1992, Pages 628-632.

[19] E. Cottereau et al., "ARAMIS: An ambidextrous 2 MV accelerator for IBA and MeV implantation", Nuclear Instruments and Methods in Physics Research Section B: Beam Interactions with Materials and Atoms, Volume 45, Issues 1-4, 2 January 1990, Pages 293-295.

[20] http://home.rzg.mpg.de/ mam/References.html 